\title{
Noncoding RNAs in the Brain
}

\author{
John S. Satterlee, ${ }^{1}$ Scott Barbee, ${ }^{2}$ Peng Jin, ${ }^{3}$ Anna Krichevsky, ${ }_{4}^{4}$ Sofie Salama, ${ }^{5}$ Gerhard Schratt, ${ }^{6}$ and Da-Yu Wu ${ }^{1}$ \\ ${ }^{1}$ Division of Basic Neuroscience and Behavioral Research, National Institute on Drug Abuse, Bethesda, Maryland 20892, ${ }^{2}$ Department of Biological Sciences, \\ University of Denver, Denver, Colorado 80208, ${ }^{3}$ Department of Human Genetics, Emory University School of Medicine, Atlanta, Georgia 30322, ${ }^{4}$ Neurology \\ Center for Neurologic Diseases, Brigham and Women's Hospital Harvard Medical School, Boston, Massachusetts 02115, ${ }^{5}$ Center for Biomolecular \\ Engineering, University of California, Santa Cruz, Santa Cruz, California 95064, and ' Interdisciplinary Center for Neurosciences, University of Heidelberg, \\ D-69120 Heidelberg, Germany
}

Cells transcribe thousands of RNAs that do not appear to encode proteins. The neuronal functions of these noncoding RNAs (ncRNAs) are for the most part not known, but specific ncRNAs have been shown to regulate dendritic spine development, neuronal fate specification and differentiation, and synaptic protein synthesis. ncRNAs have been implicated in a number of neuronal diseases including Tourette's syndrome and Fragile $\mathrm{X}$ syndrome. Future studies will likely identify additional neuronal functions for ncRNAs as well as roles for these molecules in other neuropsychiatric and neurodevelopmental disorders.

Key words: brain development; CREB; dendritic spine; development; differentiation; epigenetic; human forebrain development; learning and memory; neurogenesis; Purkinje neurons; RNA; synaptic plasticity

\section{ncRNA structure and function}

Our genomes encode thousands of RNA molecules, which come in many different varieties. The most familiar of these varieties are the mRNAs that code for protein products, but there are large numbers of transcribed RNAs that do not code for proteins and thus are referred to as noncoding RNAs (ncRNAs) (Carninci et al., 2005; Kapranov et al., 2007). Some ncRNAs, including small nuclear and small nucleolar RNAs, regulate steps in RNA biogenesis, including splicing, editing, and RNA modifications (Mehler and Mattick, 2007). ncRNAs such as tRNAs and rRNAs are involved in translation of mRNA to protein.

ncRNAs come in many sizes ranging from $\sim 21$ nucleotide molecules, including microRNAs (miRNAs), small interfering RNAs (siRNAs), Piwi-interacting RNAs, and repeat-associated siRNAs, to much longer RNA molecules, such as the $\sim 2800 \mathrm{nu}-$ cleotide HAR1F (human accelerated region 1F) or the 108,000 nucleotide AIR ncRNA, which is involved in the silencing of genomically imprinted genes (Sleutels et al., 2002; Cao et al., 2006; Pollard et al., 2006; Shamovsky and Nudler, 2006; Kapranov et al., 2007; Matera et al., 2007). Many of the more exotic RNA species have not achieved prominence until recently, and the functions of some of these molecules are not completely understood. For the remainder of this review, we will focus only on three types of ncRNAs: miRNAs, siRNAs, and HAR1F.

What are the biological functions of ncRNAs? These molecules regulate a myriad of biological processes, including developmental timing, fat metabolism, cell cycle progression, gene silencing, and programmed cell death (Berezikov and Plasterk,

Received Aug. 9, 2007; revised Sept. 10, 2007; accepted Sept. 16, 2007.

Correspondence should be addressed to John S. Satterlee, Division of Basic Neuroscience and Behavioral Research, National Institute on Drug Abuse, 6001 Executive Boulevard, Bethesda, MD 20892. E-mail: satterleej@nida.nih.gov.

DOI:10.1523/JNEUROSCI.3624-07.2007

Copyright $\odot 2007$ Society for Neuroscience $\quad$ 0270-6474/07/2711856-04\$15.00/0
2005; Cao et al., 2006; Mattick and Makunin, 2006; Mehler and Mattick, 2007). Some ncRNAs are involved in epigenetic phenomena such as genomic imprinting in which expression of particular genes or even an entire chromosome is dependent on the parental origin of the gene or chromosome. However, the precise mechanisms regulating crosstalk between the three major epigenetic regulatory pathways (ncRNAs, histone modifications, and DNA methylation) remain somewhat mysterious (Grewal and Rice, 2004; Chang et al., 2006). At least some ncRNAs are important regulators of mRNA translation (Bartel, 2004; Kosik, 2006). For example, miRNAs bind to the 3' untranslated region of target mRNAs, thereby inhibiting translation by an as yet unknown mechanism.

\section{ncRNAs in the nervous system}

Until recently, the neuronal functions of ncRNAs have been poorly characterized. Several excellent reviews describe the functions of noncoding RNAs in neurons (Cao et al., 2006; Kosik, 2006; Mehler and Mattick, 2007). Some ncRNAs are enriched in brain and may be expressed ubiquitously or in a neuronal celltype-specific manner. ncRNAs have been shown to regulate neuronal processes such as brain morphogenesis, neuronal cell fate and differentiation, and transcription of neuron-specific genes.

miRNAs, a family of small noncoding RNAs of 18-24 nucleotides, are emerging as important regulators of mRNA translation in a variety of systems, including the vertebrate nervous system (Bartel, 2004; Kosik, 2006). Recently, Gerhard Schratt and coworkers described a role for the miRNA pathway in the regulation of synapse morphology in hippocampal neurons (Schratt et al., 2006). They found that a brain-specific miRNA, miR-134, is localized to the synaptodendritic compartment and negatively regulates the size of dendritic spines, the major sites of excitatory synaptic transmission in hippocampal neurons. This effect was mediated by miR-134 inhibition of the translation of an mRNA 
encoding Limk1 (Lim kinase 1), a kinase that was known to control the dendritic spine actin cytoskeleton. Dr. Schratt and colleagues have recently embarked on a genome-wide functional screen to elucidate the full complement of miRNAs involved in dendritic spine morphogenesis.

We know a great deal about the protein machinery required for processing miRNAs and siRNAs into their functional forms (Bartel, 2004). However, there is much less known about the specific proteins that function with ncRNAs. One example of this type of protein is Drosophila $\mathrm{Me} 31 \mathrm{~B}$, which is present in neuronal and maternal RNA granules as well as cytoplasmic RNA processing bodies (P-bodies). Recently, it has been shown that Me31B is also required for dendritic morphogenesis and miRNA function in vivo (Barbee et al., 2006). A growing body of evidence indicates there is a close relationship between miRNA and P-body function (Eulalio et al., 2007; Jakymiw et al., 2007). Together, these data suggest that Me31B and other P-body components may be important factors required for ncRNA-mediated regulation of synaptic mRNA translation and long-term memory.

What is the role of ncRNAs in memory? At least two studies suggest a potential role for ncRNAs in this process. The cAMP response element-binding protein (CREB) transcription factor has been implicated in a variety of neuronal functions, including learning and memory (Carlezon et al., 2005). Recently, miR-132 has been identified as a CREB target, and overexpression of this miRNA leads to increased outgrowth of primary neurites (Vo et al., 2005). Some very exciting work in Drosophila shows that Armitage, a protein component of the RNA interference pathway, regulates both synaptic protein synthesis and long-term memory (Ashraf et al., 2006). These results suggest that miRNAs themselves could be involved in memory-related processes, although whether these are the same miRNAs that regulate dendritic spine morphogenesis remains to be seen.

\section{ncRNAs in neuronal development and brain evolution}

There have been a number of studies describing roles for ncRNAs in invertebrate and vertebrate neuronal development. One of the first was a study in the nematode Caenorhabditis elegans in which two miRNAs were found to act in a pathway regulating left/right asymmetry in the ASE chemosensory neuron pair (Chang et al., 2004). Since this initial work, this pathway been expanded to include a number of miRNA-regulated $\mathrm{Zn}$ finger and homeobox transcription factors that comprise an elaborate gene regulatory network specifying neuronal cell fates (Hobert, 2006). Anna Krichevsky and coworkers have recently shown that miRNAs play a significant role in neurogenesis in mammals. Mouse embryonic stem cells were allowed to differentiate into neuronal progenitor cells, which in turn differentiated into astrocytes or neuronal cells. Overexpression of specific miRNAs in neuronal precursor cells led to decreased numbers of astrocytes, whereas inhibition of miR-9 led to decreased numbers of neuronal cells (Krichevsky et al., 2007).

One of the most developed stories concerning noncoding RNAs and neuronal differentiation is that of miR-124, which is highly expressed in the brain. Initial work in HeLa cells indicated that overexpression of miR-124 led to decreased expression of dozens of non-neuronal genes reflective of a gene expression profile more similar to that of neuronal cells (Lim et al., 2005). Complementary studies showed that levels of miR-124 in neural progenitor cells can be reduced by the zinc-finger protein REST (repressor element-1 silencing transcription factor), enabling non-neuronal transcripts to perdure. Differentiation of neural precursor cells into adult neurons is associated with the absence of REST, increased levels of miR-124, and increased expression of neuronal genes (Conaco et al., 2006). Related work suggests that the SCP1 (small C-terminal domain phosphatase 1) phosphatase, which has an anti-neural function, is a miR-124 target. miR-124 posttranscriptionally reduces levels of the SCP1, leading to a neuronal cell fate (Visvanathan et al., 2007). The miR-124 story is not without controversy, because Cao and coworkers failed to reveal an miR-124-dependent change in neuronal fate, although they did identify endogenous miR-124 targets (Cao et al., 2007). The most recent piece in the miR-124 puzzle is the identification of the miR-124 target PTBP1, which encodes polypyrimidine tract binding protein 1 . As neurons differentiate, miR-124 reduces PTBP1 message levels, which in turn induces "a switch from general to neuron-specific alternate splicing” (Makeyev et al., 2007). Together, these studies provide valuable insight into the mechanisms by which miRNAs specify neuronal cell fates.

A particularly exciting result is the identification of an ncRNA called HAR1F that is expressed during cortical development and has evolved rapidly in humans. In humans and macaques, it is expressed in Cajal-Retzius neurons, a cell type implicated in regulation of cortical neuron migration, during early to midgestation. The $\sim 2800$ nucleotide HAR1F forms a novel stable secondary structure. Interestingly, the human-specific substitutions appear to alter a portion of this secondary structure. This ncRNA may in part contribute to differences in cortical development between humans and chimpanzees (Pollard et al., 2006). In related work, Plasterk and coworkers have identified 447 new miRNAs from human and chimpanzee brain (Berezikov et al., 2006). Many of these miRNAs appear to be primate specific and thus of more recent evolutionary origin. Together, these results suggest a possible role for ncRNAs in the regulation of primate brain development and function.

\section{ncRNAs: roles in brain disease and also therapy?}

ncRNAs have been implicated in a number of diseases of the nervous system, including gliomas, Prader-Willi syndrome, spinocerebellar ataxia type 8 , polyglutamine expansion diseases, neurodegenerative disorders, Tourette's syndrome, and Fragile X syndrome (Bilen et al., 2006; Mehler and Mattick, 2007). For example, numerous miRNAs are dysregulated in human brain malignancies, particularly in gliomas. One interesting example is miR-21, which is strongly upregulated in glioblastoma multiforme, as well as in many other human tumors, and may play an antiapoptotic role (Chan et al., 2005).

Human genetics studies have identified an association between Tourette's syndrome and variants in the SLITRK1 (SLIT and NTRK-like family, member 1) gene (Abelson et al., 2005). Overexpression of SLITRK1 in cultured cortical neurons increases dendritic growth. Interestingly, two of the gene variants identified altered an miR-189 binding site in SLITRK1. Thus, mutations altering this binding site could lead to misregulation of SLITRK1 by miR-189, subsequent alterations in dendritic growth, and ultimately Tourette's syndrome.

Recent work has identified a possible role for miRNAs in neurodegenerative disorders such as Alzheimer's or Parkinson's disease. Anne Schaefer and coworkers conditionally eliminated the Dicer protein, which is required to process miRNAs into their functional forms, specifically in postmitotic Purkinje cells in mouse cerebellum (Schaefer et al., 2007). The Purkinje neurons degenerated, suggesting that miRNAs may be required for survival of these neurons. Interestingly the mouse pups exhibited both tremor and ataxia, and the ataxia became more severe over time. The specific miRNAs that may be involved are not yet 
known. In related work, miR-133b levels were found to be reduced in midbrain samples from Parkinson's patients compared with controls (Kim et al., 2007). Functional experiments using both intact mice and embryonic stem cells suggests that this miRNA modulates the development and function of dopamine neuron, perhaps in a feedback control loop with Pitx3 (pairedlike homeodomain transcription factor 3 ). This work suggests that ncRNAs may play a role in diseases that impinge on dopamine systems, such as Parkinson's disease, schizophrenia, and addiction.

In work by Peng Jin and others, the Fragile X mental retardation protein (FMRP) was found to form a complex with the Dicer and AGO1 (Argonaute 1) proteins, both of which function in the miRNA pathway (Jin et al., 2004a,b). Interestingly, the Drosophila FMRP homolog regulates synaptic plasticity and requires AGO1 for this function. At this time, the precise role that miRNAs may play in Fragile X syndrome remains unclear. As research in this area continues, it is likely that other neurodevelopmental and neuropsychiatric diseases will be linked to ncRNAs.

The identification of ncRNAs in neuronal function and brain disease also suggests that ncRNAs may be useful targets for the development of therapeutic agents. In fact, researchers have successfully engineered molecules called antagomirs that can silence miRNAs in vivo (Krutzfeldt et al., 2005). For therapeutic agents that need to reach the CNS, the blood-brain barrier is often a significant obstacle. However researchers have recently been able to target siRNAs to the CNS. siRNAs are $\sim 22$ bp double-stranded RNAs that can silence expression of their cognate gene targets. In one experiment, an siRNA targeted against green fluorescent protein (GFP) was conjugated to a 29 amino acid acetylcholine receptor binding peptide, enabling the conjugated molecule to specifically target the CNS (Kumar et al., 2007). This molecule was able to reduce brain-specific GFP expression. A similar experiment using an siRNA targeted against a viral gene was able to protect mice against virally mediated brain inflammation. These experiments strongly suggest that siRNA inactivation of specific genes may be a useful avenue for future treatment of human diseases, including diseases of the CNS.

\section{The future of ncRNA research}

Although we now know that ncRNAs are involved in many previously unanticipated areas of neurobiology, what does the future hold for this research area? Some areas of investigation that are likely to be fruitful include the following: identification of additional dendrite-specific ncRNAs regulating synapse development and plasticity, as well as identification and functional characterization of ncRNAs expressed in specific brain regions or in response to specific pharmacological or drug treatments.

Investigations into the changes in ncRNA expression that occur during brain development may help us begin to understand what impact these molecules may have in brain development and function. The identification of ncRNA targets as well as messages that cannot be regulated by a given ncRNA (anti-targets) will help to place ncRNAs into known regulatory pathways and further delineate their biological functions. The detailed characterization of the protein machinery required for ncRNA processing and function would be of great interest. Structural and functional investigations into the zoo of subcellular RNA-containing particles (P-bodies, neuronal granules, Cajal bodies, Gems, nuclear speckles) (Lamond and Spector, 2003; Cioce and Lamond, 2005; Eulalio et al., 2007) should be very interesting. Epigenetic regulators such as histone-modifying enzymes and DNA methylases are significant players in learning and memory (Levenson and Sweatt, 2006). Do noncoding RNAs also play an important role? Finally, in the area of human health, do ncRNAs play an as yet undiscovered role in other neuropsychiatric or neurodevelopmental disorders? If so, can novel therapies be developed to target these ncRNAs and improve health outcomes?

\section{References}

Abelson JF, Kwan KY, O’Roak BJ, Baek DY, Stillman AA, Morgan TM, Mathews CA, Pauls DL, Rasin M, Gunel M, Davis NR, Ercan-Sencicek AG, Guez DH, Spertus JA, Leckman JF, Dure LS, Kurlan R, Singer HS, Gilbert DL, Farhi A, et al. (2005) Sequence variants in SLITRK1are associated with Tourette's syndrome. Science 310:317-320.

Ashraf SI, McLoon AL, Sclarsoc SM, Kunes S (2006) Synaptic protein synthesis associated with memory is regulated by the RISC pathway in Drosophila. Cell 124:191-205.

Barbee SA, Estes PS, Cziko A, Hillebrand J, Luedeman RA, Coller JM, Johnson N, Howlett IC, Geng C, Ueda R, Brand AH, Newbury SF, Wilhelm JE, Levine RB, Nakamura A, Parker R, Ramaswami M (2006) Staufen-and FMRP containing neuronal RNPs are structurally and functionally related to somatic P bodies. Neuron 52:997-1009.

Bartel DP (2004) MicroRNAs: genomics, biogenesis, mechanism, and function. Cell 116:281-297.

Berezikov E, Plasterk RH (2005) Camels and zebrafish, viruses and cancer: a microRNA update. Hum Mol Genet 14:R183-R190.

Berezikov E, Thuemmler F, Van Laake LW, Kondova I, Bontrop R, Cuppen E, Plasterk RH (2006) Diversity of microRNAs in human and chimpanzee brain. Nat Genet 38:1375-1377.

Bilen J, Liu N, Burnett BG, Pittman RN, Bonini NM (2006) MicroRNA pathways modulate polyglutamine-induced neurodegeneration. Mol Cell 24:157-163.

Cao X, Yeo G, Muotri AR, Kuwabara T, Gage FH (2006) Noncoding RNAs in the mammalian central nervous system. Annu Rev Neurosci 29:77-103.

Cao X, Pfaff SL, Gage FH (2007) A functional study of miR-124 in the developing neural tube. Genes Dev 21:531-536.

Carlezon WA, Duman RS, Nestler EJ (2005) The many faces of CREB. Trends Neurosci 28:436-445.

Carninci P, Kasukawa T, Katayama S, Gough J, , Maeda N, , Ravasi T, Lenhard B, Wells C, Kodzius R, Shimokawa K, Bajic VB, Brenner SE, Batalov S, Forrest AR, Zavolan M, Davis MJ, Wilming LG, Aidinis V, et al. (2005) The transcriptional landscape of the mammalian genome. Science 309:1559-1563.

Chan JA, Krichevsky AM, Kosik KS (2005) MicroRNA-21 is an antiapoptotic factor in human glioblastoma cells. Cancer Res 65:6029-6033.

Chang S, Johnston RJ, Frekjaer-Jensen C, Lockery S, Hobert O (2004) MicroRNAs act sequentially and asymmetrically to control chemosensory laterality in the nematode. Nature 430:785-789.

Chang SC, Tucker T, Thorogood NP, Brown CJ (2006) Mechanisms of $\mathrm{X}$-chromosome inactivation. Front Biosci 11:852-866.

Cioce M, Lamond AI (2005) Cajal bodies: a long history of discovery. Annu Rev Cell Dev Biol 21:105-131.

Conaco C, Otto S, Han JJ, Mandel G (2006) Reciprocal actions of REST and a microRNA promote neuronal identity. Proc Natl Acad Sci USA 103:2422-2427.

Eulalio A, Behm-Ansmant I, Izaurralde E (2007) P bodies: at the crossroads of post-transcriptional pathways. Nat Rev Mol Cell Biol 8:9-22.

Grewal SI, Rice JC (2004) Regulation of heterochromatin by histone methylation and small RNAs. Curr Opin Cell Biol 16:230-238.

Hobert O (2006) Architecture of a microRNA- controlled gene regulatory network that diversifies neuronal cell fates. Cold Spring Harb Symp Quant Biol 71:181-188.

Jakymiw A, Pauley KM, Li S, Ikeda K, Lian S, Eystathioy T, Satoh M, Fritzler NJ, Chan EK (2007) The role of GW/P-bodies in RNA processing and silencing. J Cell Sci 120:1317-1323.

Jin P, Zarnescu DC, Ceman S, Nakamoto M, Mowrey J, Jongens TA, Nelson DL, Moses K, Warren ST (2004a) Biochemical and genetic interaction between the fragile X mental retardation protein and the microRNA pathway. Nat Neurosci 7:113-117.

Jin P, Alisch RS, Warren ST (2004b) RNA and microRNAs in Fragile X mental retardation. Nat Cell Biol 6:1048-1053.

Kapranov P, Cheng J, Dike S, Nix DA, Duttagupta R, Willingham AT, Stadler 
PF, Hertel J, Hackermuller J, Hofacker IL, Bell I, Cheung E, Drenkow J, Dumais E, Patel S, Helt G, Ganesh M, Ghosh S, Piccolboni A, Sementchenko V, Tammana H, Gingeras TR (2007) RNA maps reveal new RNA classes and possible function for pervasive transcription. Science 316:1484-1488.

Kim J, Inoue K, Ishii J, Vanti WB, Voronov SV, Murchison E, Hannon G, Abeliovich A (2007) A microRNA feedback circuit in midbrain dopamine neurons. Science 317:1220-1224.

Kosik KS (2006) The neuronal microRNA system. Nat Rev Neurosci 7:911-920.

Krichevsky AM, Sonntag K, Isacson O, Kosik KS (2007) Specific MicroRNAs modulate embryonic stem cell derived neurogenesis. Stem Cell 24:857-866.

Krutzfeldt J, Rajewsky N, Braich, Rajeev KG, Tuschi T, Manoharan M, Stoffel M (2005) Silencing of microRNAs in vivo with "antagomirs." Nature 4381:685-689.

Kumar P, Wu H, McBride JL, Jung K, Kim MH, Davidson BL, Lee SK, Shankar P, Manjunath N (2007) Transvascular delivery of small interfering RNA to the central nervous system. Nature 448:39-43.

Lamond AI, Spector DL (2003) Nuclear speckles: a model for nuclear organelles. Nat Rev Mol Cell Biol 4:605-612.

Levenson JM, Sweatt JD (2006) Epigenetic mechanisms: a common theme in vertebrate and invertebrate memory formation. Cell Mol Life Sci 63:1009-1016.

Lim LP, Lau NC, Garrett-Engele P, Grimson A, Schelter JM, Castle J, Bartel DP, Linsley PS, Johnson JM (2005) Microarray analysis shows that some microRNAs downregulate large numbers of target mRNAs. Nature 433:769-773.

Makeyev EV, Zhang J, Carrasco MA, Maniatis T (2007) The microRNA muR-124 promotes neuronal differentiation by triggering brain-specific alternative pre-mRNA splicing. Mol Cell 27:435-448.
Matera AG, Terns RM, Terns MP (2007) Non-coding RNAs: lessons from the small nuclear and small nucleolar RNAs. Nat Rev Mol Cell Biol 8:209-220.

Mattick JS, Makunin IV (2006) Non-coding RNA. Hum Mol Genet 15:R17-R29.

Mehler MF, Mattick JS (2007) Non-coding RNAs and RNA editing in brain development, functional diversification, and neurological disease. Physiol Rev 87:799-823.

Pollard KS, Salama SR, Lambert N, Lambot M, Coppens S, Pedersen JS, Katzman S, King S, King B, Onodera C, Siepel A, Kern AD, Dehay C, Igel H, Ares Jr M, Vanderhaeghen P, Haussler D (2006) An RNA gene expressed during cortical development involved rapidly in humans. Nature 443:167-172.

Schaefer A, O'Carroll D, Tan CL, Hillman D, Sugimori M, Llinas R, Greengard P (2007) Cerebellar neurodegeneration in the absence of microRNAs. J Exp Med 204:1553-1558.

Schratt GM, Tuebing F, Nigh EA, Kane CG, Sabatini ME, Kiebler M, Greenberg ME (2006) A brain-specific microRNA regulates dendritic spine development. Nature 439:283-289.

Shamovsky I, Nudler E (2006) Gene control by large noncoding RNAs. Sci STKE:pe39.

Sleutels F, Zwart R, Barlow DP (2002) The non-coding Air RNA is required for silencing autosomal imprinted genes. Nature 415:810-813.

Visvanathan J, Lee S, Lee B, Lee JW, Lee SK (2007) The microRNA miR-124 antagonizes the anti-neural REST/SCP1 pathway during embryonic CNS development. Genes Dev 21:744-749.

Vo N, Klein ME, Varlamova O, Keller DM, Yamamoto T, Goodman RH, Impey S (2005) A cAMP-response element binding protein-induced microRNA regulates neuronal morphogenesis. Science 102: $16426-16431$. 